\title{
Study on mechanical behaviors of articular cartilage defect repaired area under cyclic compression loading.
}

\author{
Xi-Ge Ma ${ }^{1,2}$, Hai-Ying Liu ${ }^{1,2 *}$, Cheng-Fei Du ${ }^{1,2}$, Wei Wang ${ }^{3}$, Chun-Qiu Zhang ${ }^{1,2 *}$ \\ ${ }^{1}$ Tianjin Key Laboratory for Advanced Mechatronic System Design and Intelligent Control, School of Mechanical \\ Engineering, Tianjin University of Technology, Tianjin, 300384, P.R. China \\ ${ }^{2}$ National Demonstration Center for Experimental Mechanical and Electrical Engineering Education, Tianjin University \\ of Technology, Tianjin, 300384, P.R. China
}

${ }^{3}$ Department of Mechanics, School of Mechanical Engineering, Tianjin University, Tianjin, 300354, P.R. China

\begin{abstract}
In this paper, the mechanical behaviors of the articular cartilage defect repaired area were studied under cyclic compression loading, and also discussed the material parameters index of tissue engineered cartilage (TEC) and loading frequency might prove beneficial. A dual-phase fiber-reinforced porous elastic model of articular cartilage was established by finite element software COMSOL, in which depthdependent mechanical parameters of cartilage was considered. The stress distribution in the repaired area, the displacement difference between two adjacent paths on both sides of the repaired interface and the stress respond of the solid matrix to the cyclic loading was studied numerically. The mechanical environment of the repaired area changed significantly. At the same frequency, the TEC with lower elastic modulus (0.1 MPa, 0.3 MPa), the maximum value of stress and the displacement difference between adjacent paths were lower in the repaired area. The peak-peak value of stress respond curves of solid matrix to cyclic loading at higher frequency $(0.5 \mathrm{~Hz})$ was lower than that at lower frequency $(0.1$ $\mathrm{Hz}$ ) in each layer. According to the numerical simulation results in this paper, the TEC with elastic modulus $(0.3 \mathrm{Mpa})$ and the cyclic loading at frequency $(0.5 \mathrm{~Hz})$, the mechanical environment of the repaired area was better than other conditions. This work has some guiding significance for the development of biomedical basis and patients in clinical care and rehabilitation training technology.
\end{abstract}

Keywords: Cyclic loading, Defect repairing, Numerical simulation, Stress respond.

Accepted on February 22, 2018

\section{Introduction}

Articular cartilage is a mechanical adapted tissue without blood vessels, lymph, and nerves, and often endures complex stress environment under physiological loading. TEC as substitute material holds great promise to repair, regenerate, and/or improve injured or diseased articular cartilage functionality without the shortcomings. In the past decades, enormous progress has been made in the optimization of strategies for cartilage tissue engineering, which has been used in clinical treatment, but to date, there are still many issues that need to be researched. For example, Cartilage repair often results in fibrocartilaginous tissue without the production of normal hyaline cartilage [1-3]. The mechanical environment has great effects on the maintenance, growth and development of cartilage. It is of great theoretical significance and practical value for researching the mechanical behaviors of the articular cartilage defect repaired area after implanting TEC during the treatment of joint diseases, clinical diagnosis and the development of artificial joint materials [4-6].
Many experimental references suggested the effects of different material characteristics on osteochondral defects repair [7-10]. Frenkel et al. studied the restorative effects of two TECs with different material characteristics on osteochondral defects in rabbits, and the results showed that the toughness of the host cartilage and the hardness of the subchondral bone were changed after implantation of TEC [8]. Chen et al. found that if full-thickness defect of rabbit articular cartilage was given exercise intervention, and it could promote tissue repairing and synthesis of type II collagen and proteoglycan, but we still have not known the mechanobiology and biomechanics of the articular cartilage defect repaired area [9].

There are few numerical analysis on the mechanical behaviors of the articular cartilage defect repaired area after implanting, although several computational models have been established to investigate cartilage modeling and remodelling [11-13]. In view of the complexity of stress loads and the diversity of motion patterns in joint motion, it is difficult that the 
experimental studies on stress environment of cartilage be carried out in vivo. The numerical simulation is a good method studing on the mechanical behaviors of articular cartilage. In 1980, Mow proposed the theoretical model of cartilage twophase porous medium for the first time, and established a precedent in calculating cartilage interstitial flow. The model successfully described the mechanical behaviors of cartilage, and opened the door to study of cartilage physiology and mechanical biology [10]. Wang et al. established a dual-phase model of cartilage, and the strain of solid matrix was studied by numerical simulation under cyclic compressive loading, and the effect of mechanical stimulation on arthritis was investigated at the microscopic cellular level [14].

In this paper, the solid-liquid coupling biphasic mechanical model of the articular cartilage was established by the finite element software COMSOL. According to the curve contact characteristics of the femur and tibia surface in the knee joint, so the physiological load conformed to the Hertz distribution law was applied to the articular surfaces. The frequencies of cyclic compressive loading were $0.1 \mathrm{~Hz}$ and $0.5 \mathrm{~Hz}$ respectively, a comparative study on the mechanical behaviors of the defect repaired area was conducted by numerical simulation. Through comparing the stress distribution of defect repaired area, displacement difference between adjacent paths on both sides of adhesion interface, the stress respond characteristics of the solid matrix, to find out suitable parameters to assist clinical treatment.

\section{Materials and Methods}

\section{Modeling and material parameters setting}

In daily activities, the contact stress of human knee joints varies widely, such as walking, riding and climbing stairs, the articular cartilage must bear a load equal to $6 \sim 10$ times the body weight [15]. Usually, stride frequency of the human being is about 95 to 125 steps per min, that is, the frequency is 0.48 $\mathrm{Hz}$ to $0.63 \mathrm{~Hz}$. In this paper, sinusoidal load was applied to the joint surface to simulate physiological loads during rehabilitation exercise. The frequencies of cyclic loading were $0.1 \mathrm{~Hz}$ and $0.5 \mathrm{~Hz}$, the amplitude was $2000 \mathrm{~N}$, which was equivalent to the force exerted on the joints during the walking of a person who weighed around $65 \mathrm{~kg}$.

The length and the thickness of the cartilage model were 20 $\mathrm{mm}$ and $2 \mathrm{~mm}$, the length and the depth of the middle layer defect were $2 \mathrm{~mm}$ and $0.7 \mathrm{~mm}$ (Figure 1), and the TEC with elastic modulus( $0.1 \mathrm{MPa}, 0.3 \mathrm{MPa}, 0.6 \mathrm{MPa}$, and $0.9 \mathrm{MPa})$. According to the difference of the content of the solid matrix and mechanical properties in each layer, the articular cartilage is divided into the superficial layer, middle layer and deep layer [16].

The depth-dependent material parameters of articular cartilage, for instance, the elastic modulus (E), permeability $(\mathrm{k})$, and Poisson's ratio (v) were obtained by a higher number of experiments, which improved the theoretical model of articular cartilage $[4,17-20]$.

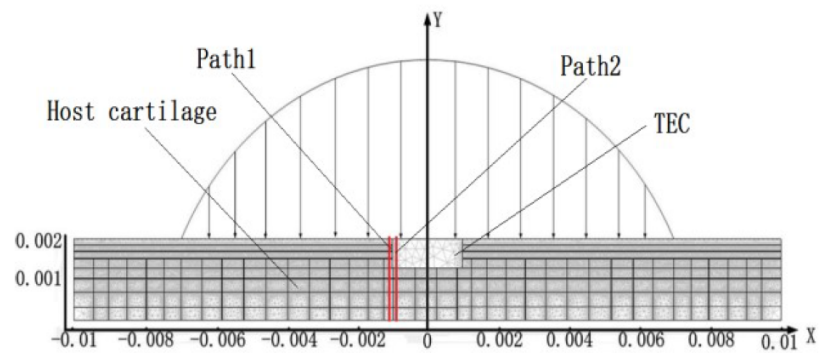

Figure 1. Model of articular cartilage defect and boundary pressure.

\section{Reliability verification of cartilage model}

After the continued efforts of many scholars, to establish the mechanical model of articular cartilage has undergone the process from single-phase to three-phase, isotropic to anisotropic, linear elastic to hyper elastic and viscoelastic. The structural features of cartilage were considered one by one, the model was also getting closer to native cartilage [19]. There are three common ways to measure mechanical behaviors of cartilage by experimental method, for example, confined compression, unconfined compression and indentation test. In this paper, a cartilage model with fixed bottom and laterally unconfined was established, which was consistent with the experimental conditions of unconfined compression and closer to the physiological environment of articular cartilage.

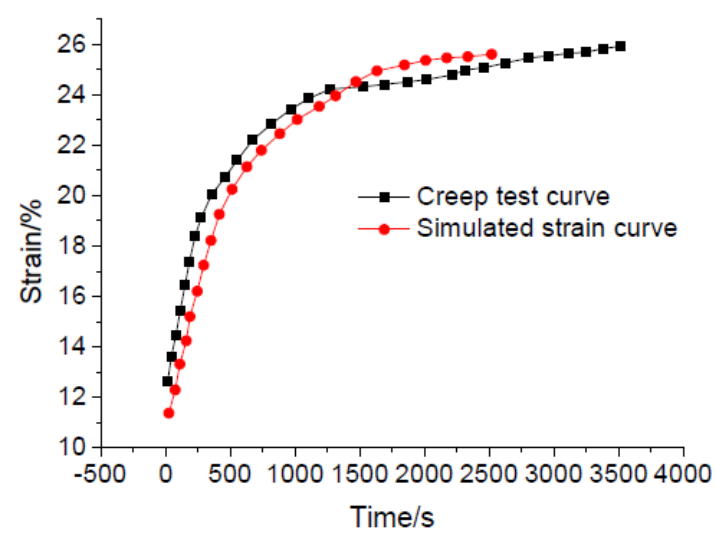

Figure 2. The simulation curve in this paper and test curve in the literature [21].

In 2003, Meng et al. made a study on the creep test of human articular cartilage, loaded at a uniform stress acceleration until the strain reached $12.6 \%$, at this point, the stress reached 2.75 $\mathrm{MPa}$ and remained constant, after that, strain increased with time [21]. In this paper, the creep condition of the cartilage model was studied by numerical simulation, applied the same loading on cartilage surface as the literature [21]. The creep curve of the cartilage finite element model in this paper was compared with that of the cartilage creep test in the literature, as shown in Figure 2, so you can see that the results obtained by the two methods were in good agreement [21]. It showed that the model in this paper was reasonable, and could guarantee the validity of the follow-up analysis. 
In recent years, with the development of tissue engineering technology, it hopefully will be a method to cure cartilage injuries, while the factors that influence the final restorative effect in clinic has become one of the hot issues of research.

\section{Results}

\section{The stress contours of the cartilage defect repaired area}

The stress contours of cartilage defect repaired area under cyclic loading at frequencies $(0.1 \mathrm{~Hz}, 0.5 \mathrm{~Hz})$ were obtained, as shown in Figure 3. The difference of mechanical properties between TEC and the host cartilage resulted in stress concentration at the repaired boundary, for TEC with various elastic modulus (0.1 Mpa, 0.3 Mpa, 0.6 Mpa, 0.9 Mpa). In addition, the cartilage is fixed on the subchondral bone, owing to the subchondral bone limits the tangential displacement of the cartilage, and stress concentration also appeared in this area. The TEC with lower elastic modulus $(0.1 \mathrm{MPa}, 0.3 \mathrm{MPa})$, the maximum stress appeared on both sides of the vertical adhesive interface. The TEC with higher elastic modulus $(0.6$ $\mathrm{MPa}, 0.9 \mathrm{MPa}$ ), the maximum stress appeared at bottom side of the adhesive interface. By comparing the maximum stress in a variety of situations, TEC with lower elastic modulus $(0.3$ $\mathrm{Mpa})$, and the cyclic loading at higher frequency $(0.5 \mathrm{~Hz})$, the mechanical environment of the defect repaired area was better than other situations.

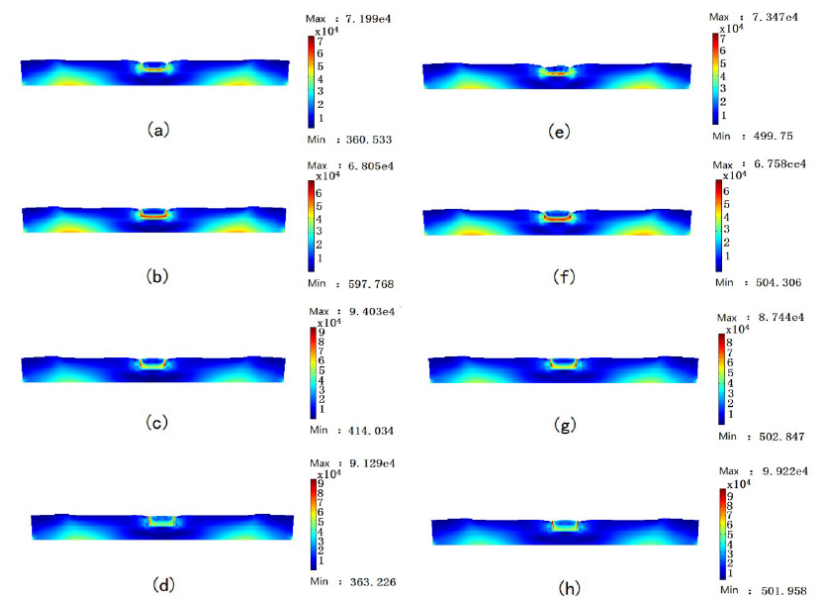

Figure 3. The stress contours of defect repaired area. (a-d) Frequency $(0.1 \mathrm{~Hz})$, and TEC with elastic modulus (0.1 MPa, 0.3 MPa, 0.6 MPa, $0.9 \mathrm{MPa})$; (e-h) Frequency $(0.5 \mathrm{~Hz})$ and TEC with elastic modulus (0.1 MPa, 0.3 MPa, 0.6 MPa, 0.9 MPa).

\section{The displacement difference between two paths on the both sides of vertical adhesive interface}

Clinically, medical biological glue can be used to glue TEC to the host cartilage for repairing the defect, but the long-term restorative effect is uncertain, that is, adhesive interface cracking and degeneration of the host cartilage has become commonplace. In this paper, the displacement difference between two paths on both sides of the defect repaired interface (the path 1 in the host cartilage, the path 2 in TEC, as shown in Figure 1) was studied, to predict the reasons of cracking at adhesive interface.

For the TEC with lower elastic modulus $(0.1 \mathrm{MPa}, 0.3 \mathrm{MPa})$, it was known from the comparison that the displacement difference between two paths at higher frequency $(0.5 \mathrm{~Hz})$ was less than that at lower frequency $(0.1 \mathrm{~Hz})$, as shown in Figures $4 \mathrm{a}$ and $4 \mathrm{~b}$. For the TEC with higher elastic modulus (0.6 MPa, $0.9 \mathrm{MPa})$, the displacement difference between two paths in the superficial layer significantly increased under cyclic loading at frequency $(0.1 \mathrm{~Hz}, 0.5 \mathrm{~Hz})$, as shown in Figures $4 \mathrm{c}$ and $4 \mathrm{~d}$, that is, the vertical adhesive interface between host cartilage and TEC may crack from top to bottom. By contrast, if the TEC with lower elastic modulus $(0.3 \mathrm{Mpa})$ was used to repair the cartilage defect, and the applied cyclic loading at higher frequency $(0.5 \mathrm{~Hz})$, which was beneficial to defect repairing
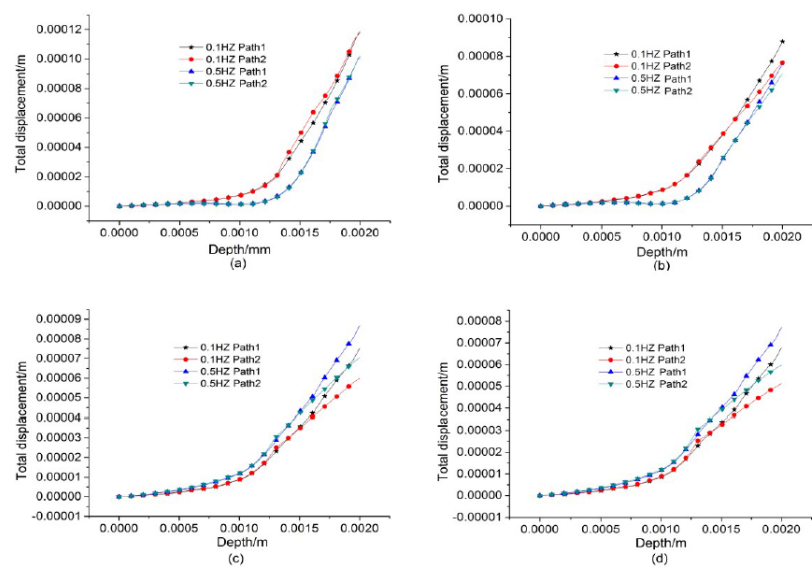

Figure 4. Displacement of two paths under cyclic loading at frequency $(0.1 \mathrm{~Hz}, 0.5 \mathrm{~Hz})$. (a-d) TEC with elastic modulus $(0.1 \mathrm{MPa}$, $0.3 \mathrm{MPa}$, 0.6 MPa and 0.9 MPa respectively).

\section{Stress respond of solid matrix to the cyclic loading}

The destruction of cartilage solid matrix is the macroscopic expression of its micro-structure damage. The main reason for cartilage degeneration is that the mechanical environment of chondrocytes embedded in solid matrix beyond the range of physiological loads, which causes chondrocytes to fail to normal gene expression, even damage and destruction. As a result, articular cartilage can't maintain its mechanical properties and the topological construction, ultimately, result in cartilage becoming vestigial.
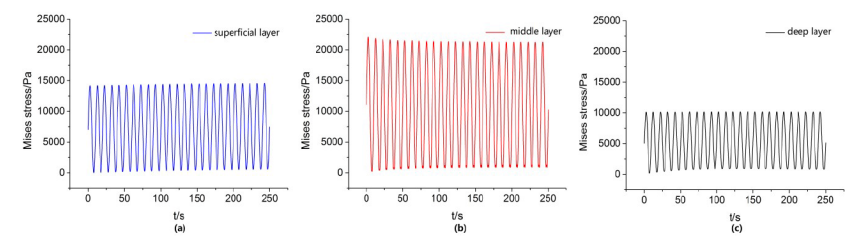

Figure 5. The stress respond curves of intact cartilage to cyclic loading at frequency $(0.1 \mathrm{~Hz})$ with time. (a) Superficial layer; (b) Middle layer; (c) Deep layer.

Owing to the mechanical properties of TEC are not comparable to those of natural cartilage, the mechanical environment of the 
defect repaired area was changed significantly. Stress respond curves of each layer solid matrix to cyclic loading, which could be quantitatively studied by taking a point in the superficial layer, middle layer and deep layer of the host cartilage, respectively. Generally speaking, the stress concentration in the adjacent area of adhesive interface was more pronounced, so the selected three points was very close to interface and on a vertical line. The curves of stress respond with time were obtained by numerical simulation, as shown in Figures 5 and 6 . In this paper, to study the difference of stress respond law between intact cartilage and defect repaired cartilage to cyclic compression loading.
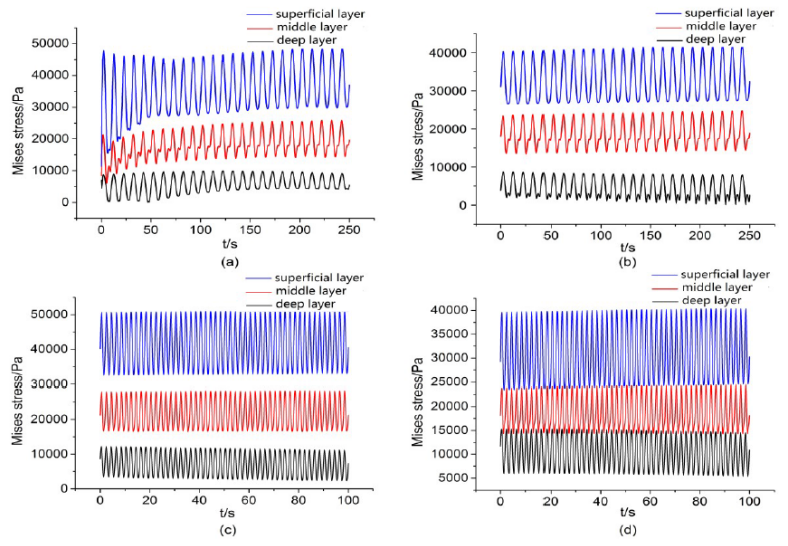

Figure 6. The stress respond curves of each layer host cartilage of defect repaired area with time. $(a, b)$ Frequency $(0.1 \mathrm{~Hz})$, the TEC with elastic modulus (0.1 MPa, $0.3 \mathrm{MPa})$; (c, d) Frequency $(0.5 \mathrm{~Hz})$, the TEC with elastic modulus (0.1 MPa, 0.3 MPa).

For the intact cartilage, the stress respond curves of each layer solid matrix varied periodically with the time, owing to the peak-peak values of the stress respond curves were extremely close at two frequencies $(0.1 \mathrm{~Hz}, 0.5 \mathrm{~Hz})$, so only the stress respond curves at frequency $(0.1 \mathrm{~Hz})$ was given, as shown in Figure 5, in this paper. The peak-peak values of the steadystate responds curves of superficial layer, middle layer and deep layer were $0 \sim 14.175 \mathrm{KPa}, 0 \sim 22.103 \mathrm{KPa}, 0 \sim 10.175 \mathrm{KPa}$, respectively. It was obvious that the upper peak value of stress respond curve of the middle layer was highest, and lower peak value was 0 for each layer. The frequency of cyclic loading has little influence on the peak-peak values of the stress respond curves of the intact cartilage.

The TEC with lower elastic modulus $(0.1 \mathrm{MPa}, 0.3 \mathrm{MPa})$, the mechanical environment of the defect repaired area was better from previous studies. The stress respond curves of each layer of the host cartilage with time, as shown in Figure 6 . Compared with the intact cartilage, the stress respond curves of each layer to the cyclic loading changed significantly after the defect repairing, the peak-peak values of stress respond curves in each layer increased. The peak-peak value of stress respond curves of the superficial layer was greater than that of the middle layer, and increased significantly. For the middle layer, the upper peak values increased less, and lower peak values increased by a higher margin. The peak-peak values increased within a narrow range for the deep layer. Percentages of the upper peak values of stress respond curves of the defect repaired cartilage and the intact cartilage were listed in Table 1. In conclusion, the TEC with elastic modulus $(0.3 \mathrm{Mpa})$, the stress distribution in the repaired was more reasonable for superficial layer defect repairing.

Table 1. Percentages of the maximum values of stress respond curve.

\begin{tabular}{lllll}
\hline Load frequency (Hz) & $\mathbf{0 . 1}$ & $\mathbf{0 . 5}$ \\
\hline Elastic modulus of TEC (Mpa) & 0.1 & 0.3 & 0.1 & 0.3 \\
\hline Superficial layer (\%) & 344.4 & 295.7 & 357.7 & 288.8 \\
\hline Middle layer (\%) & 116.8 & 112 & 126.8 & 111.3 \\
\hline Deep layer (\%) & 89.7 & 106.6 & 101.3 & 143.8 \\
\hline
\end{tabular}

\section{Discussion}

In this paper, the mechanical behaviors of the articular cartilage defect repaired area under cyclic compression loading were studied by numerical simulation. The results showed that, at the same loading frequency, elastic modulus of TEC has a significant influence on the mechanical environment of the defect repaired area.

The stress concentration phenomenon appeared at the bottom of the adhesive surface for the TEC with lower elastic modulus (0.1 MPa, 0.3 MPa). There was serious stress concentration on both sides of the vertical adhesive interface, and obvious displacement differences between two paths close to vertical adhesive interface in superficial layer, for the TEC with higher elastic modulus (0.6 MPa, 0.9 MPa); this was also the main reason for adhesive interface cracking and TEC peeling off from host cartilage. After TEC implantation, the immediate mechanical behaviors of the cartilage defect repaired area under static compressive loading was investigated in vitro, the stress concentration at the interface was found [22]. Usually, the tissue deformation is less than $20 \%$ under physiological cyclic loading; it is believed that the cartilage will not cause substantial damage [10]. The deformation of the solid matrix can also reflect the carrying capacity of the cartilage to some extent, the displacement difference between two paths (path 1 in the host cartilage, path 2 in the TEC) close to the vertical adhesive interface was studied under cyclic compressive loading, in this paper, for the first time, to reveal factors that caused repair boundary cracking from the elastic modulus of TEC, frequency of cyclic loading, etc.

In addition, after implanting TEC, appropriate cyclic compressive loading stimulation is beneficial to the maintenance of physiological function of articular cartilage. The synthesis of cartilage collagen was impaired with the increase of frequency of cyclic loading. The cyclic loading at frequency $(1.0 \mathrm{~Hz})$, the cartilage was loaded 3600 times, Clements et al. deduced that collagen was weakened with an increase of cartilage inhibitors, furthermore, the ability of cartilage to restore its original configuration was attenuated as the cyclic loading frequency increased [23]. Nuclear factor kappa $\mathrm{B}(\mathrm{NF}-\mathrm{kB})$ is involved in acute and chronic inflammatory respond by modulating numerous pro- 
inflammatory and anti-inflammatory factors, Madhavan et al. believed that cyclic stress stimulation can protect cartilage by inhibiting nuclear factor NF kappa B transcriptional activity [24]. If the frequency of cyclic loading in an appropriate range, it is beneficial to cartilage growth and defect repairing. In this paper, comparative study on the mechanical behaviors of the defect repaired area under cyclic loading at frequencies $(0.1$ $\mathrm{Hz}, 0.5 \mathrm{~Hz}$ ) was made. The results showed that some parameters index such as stress field, displacement difference between two paths close to the vertical adhesive interface and stress respond of solid matrix in each layer under loading at frequency $(0.5 \mathrm{~Hz})$ were better than that at frequency $(0.1 \mathrm{~Hz})$. $0.5 \mathrm{~Hz}$ is in the range of human body walking at frequency $(0.48 \mathrm{~Hz} \sim 0.63 \mathrm{~Hz})$, so after defect repairing, the patient should exercise moderately, a better mechanical environment can be obtained in the repaired area.

The mechanical behaviors of the articular cartilage defect repaired area still need experimental research after implanting TEC, although the numerical simulations of defect repaired area were studied by FEM. The mechanical behaviors of defect repaired area can be researched by digital image correlation technology, which can obtain the strain distribution of defect repaired area after implanting TEC. Digital image correlation technology can recognize the changed images by noise removal using adaptive filter, segmentation using region growing algorithm and genetic algorithm with fuzzy classifier, which will verify the numerical simulation results and the laws of strain distribution within the defect repair area in the future research [25-28].

There were still some shortcomings in this paper. The location of the points in each layer was relatively extensive, which has certain influence on the research. Only middle layer defect repairing was considered, clinically, there are also superficial layer and deep layer defect repairing. Furthermore, only the cyclic loading at two frequencies was studied, and less than 0.1 $\mathrm{Hz}$ and greater than $0.5 \mathrm{~Hz}$ need further study.

\section{Conclusion}

The mechanical behaviors of the articular cartilage repaired area are one of the main factors that affect the clinical repairing effect. The change of the mechanical behaviors of the defect repaired area was mainly relating to the properties of TEC materials. In addition, the frequency of cyclic loading also affects the repairing effect to a certain extent. The mechanical behaviors of middle layer defect repaired area were studied in this paper, such as the stress field, the displacement difference of adjacent two paths and the stress respond of each layer.

By comparing the results of data analysis, it can be found that the TEC with elastic modulus $(0.3 \mathrm{Mpa})$ and the cyclic loading at frequency $(0.5 \mathrm{~Hz})$ was more available can benefit defect repairing. Therefore, the loading at moderate frequency can be obtained by daily exercise, which can create a favorable mechanical environment for restoration. The results of this study have certain guiding significance for clinical cartilage defects repairing.

\section{Acknowledgement}

The authors are grateful for the support of the National Natural Science Foundation of China (grant numbers: 11402172, 11432016, 11672208 and 11602172).

\section{Competing Interest}

The authors declare that they have no competing interests.

\section{References}

1. Zhou HY. Biotribological mechanism of articular cartilage. Shanghai: Shanghai Jiao Tong Univ 2013.

2. Sheng SH, Li XP. Stress load and articular cartilage injury. Chin J Rehab Med 2013; 28: 475-478.

3. Grad S, Eglin D, Alini M. Physical stimulation of chondrogenic cells in vitro: a review. Clin Orthop Related Res 2011; 469: 2764-2772.

4. Wang HB, Liu DX, Ren ZJ. Selection and biomechanical features of repair materials for exercise-induced articular cartilage injury. J Clin Rehab Tissue Eng Res 2011; 15: 03-19.

5. Venäläinen MS, Mononen ME, Salo J. Quantitative evaluation of the mechanical risks caused by focal cartilage defects in the knee. Sci Rep 2016; 6: 37538.

6. Accardi MA, Dini D, Philippa MC. Experimental and numerical investigation of the behaviour of articular cartilage under shear loading-Interstitial fluid pressurization and lubrication mechanisms. Tribol Int 2011; 44: 565-578.

7. Zhang RY, Lin XT, Liu PZ. Nursing care of articular cartilage defects treated with autologous chondrocytes and collagen type I scaffold. J Pract Orthop 2016; 12: 1150-1152.

8. Frenkel SR, Bradica G, Brekke JH. Regeneration of articular cartilage-evaluation of osteochondral defect repair in the rabbit using multiphasic implants. Osteoarthritis Cartilage 2005; 13: 798-807.

9. Chen WX, Zhang CJ. Effect of continuous passive motion on the healing of experimental full-thickness defects of articular cartilage in knee of rabbit. Chin J Phys Med Rehabil 2004; 26: 644-647.

10. Buschmann MD, Kim YJ, Wong M. Stimulation of aggrecan synthesis in cartilage explants by cyclic loading is localized to regions of high interstitial fluid flow. Archiv Biochem Biophys 1999; 366: 1-7.

11. Mow VC, Kuei SC, Lai WM. Biphasic creep and stress relaxation of articular cartilage in compression: Theory and experiments. J Biomech Eng 1980; 102: 73-84.

12. Korhonen RK, Laasanen MS, Toyras J. Fibril reinforced poroelastic model predicts specifically mechanical behavior of normal, proteoglycan depleted and collagen degraded articular cartilage. J Biomech 2003; 36: 1373-1379.

13. Leping LI, Soulhat J, Buschmann MD. Nonlinear analysis of cartilage in uncontained ramp compression using a fibril reinforced poroelastic model. Clin Biomech 1999; 14: 673-682. 
14. Wang X, Ayati BP, Brouillete MJ. Modeling and simulation of the effects of cyclic loading on articular cartilage lesion formation. Int J Numer Methods Biomed Eng 2015; 30: 927-941.

15. Julkunen P. Relationships between structure composition and function of articular cartilage. Univ of Kuopio 2008.

16. Liu ZD, Gao LL, Zhang CQ. Loading rate-dependent property of different layers for articular cartilage. J Med Biomech 2014; 29: 141-145.

17. Zhou HY, Li YC, Wang CT. Reinforcement property of collagen fibril in articular cartilage. J Med Biomech 2013; 28: 460-465.

18. Hosoda N, Sakai N, Sawae Y. Finite element analysis of articular cartilage model considering the configuration and biphasic property of the tissue. IFMBE Proceedings 2009; 23: $1883-1887$.

19. Duan HT, Liu HY, Wang W. Study on the effect of artificial cartilage with different elastic modulus on the mechanical environment of the chondrocyte in defect cartilage repaired area. J Biomed Eng 2017; 34: 34-40.

20. Li LP, Buschmann MD, Shiraziadl A. A fibril reinforced nonhomogeneous poroelastic model for articular cartilage: inhomogeneous respond in unconfined compression. J Biomech 2000; 33: 1533-1541.

21. Meng GW, Cheng JP, Ma HS. Experimental study on the pulling stress relaxation and creep test of patella cartilage. J Med Biomech 2003; 18: 239-255.

22. Men YT, Liu HY, Li J. Mechanical properties of instantly repaired articular cartilage defects by tissue engineering. J Med Biomech 2013; 28: 195-200.

23. Wu, Bruce YC. Investigation of long-term cyclic loading effects on initially intact cartilage. Massachusetts Institute of Technology 2007.

24. Madhavan S, Anghelina M, Sjostrom D. Biomechanical signals suppress TAK1 activation to inhibit NF-kappaB transcriptional activation in fibrochondrocytes. J Immunol 2007; 179: 6246-6254.

25. Kaluri R, Reddy CP. A framework for sign gesture recognition using improved genetic algorithm and adaptive filter. Cogent Eng 2016; 3: 1251730.

26. Kaluri R, Lakshmanna K, Reddy T, Karnam S, Koppu S. A comparative study on selecting and ranking the test cases in software testing. ARPN J Eng Appl Sci 2016; 11: 754-757.

27. Rishab Jain C, Kaluri R. Design of automation scripts execution application for selenium web driver and test $\mathrm{NG}$ framework. ARPN J Eng Appl Sci 2015; 10: 2440-2445.

28. Kaluri R, Reddy P. Sign gesture recognition using modified region growing algorithm and adaptive genetic fuzzy classifier. Int J Intell Eng Syst 2016; 9: 225-233.

\section{*Correspondence to}

Hai-Ying Liu

National Demonstration Center for Experimental Mechanical and Electrical Engineering Education

Tianjin University of Technology

Tianjin

P.R. China

Chun-Qiu Zhang

National Demonstration Center for Experimental Mechanical and Electrical Engineering Education

Tianjin University of Technology

Tianjin

P.R. China 